\title{
A LEGENDRE-BASED DISPLACEMENT FIELD FOR TWO-DIMENSIONAL DIGITAL IMAGE CORRELATION
}

\section{KEVIN T. L. M. SOUZA ${ }^{1}$, CHRISTIANO A. F. VÁRADY FILHO ${ }^{2}$ AND ROMILDO S. ESCARPINI FILHO ${ }^{3}$}

\author{
${ }^{1}$ Center of Technology - Federal University of Alagoas \\ Av. Lourival Melo Mota, S/N, Tabuleiro do Martins, 57072-970, Alagoas/Maceió, BRAZIL \\ kevin.tenorio98@gmail.com
}

${ }^{2}$ Laboratory of Scientific Computing and Visualization - Federal University of Alagoas Av. Lourival Melo Mota, S/N, Tabuleiro do Martins, 57072-970, Alagoas/Maceió, BRAZIL cafvf2@gmail.com

${ }^{3}$ Laboratory of Scientific Computing and Visualization - Federal University of Alagoas Av. Lourival Melo Mota, S/N, Tabuleiro do Martins, 57072-970, Alagoas/Maceió, BRAZIL romildo@lccv.ufal.br

Key words: Displacement Field, Digital Image Correlation

\begin{abstract}
Two-dimensional digital image correlation (DIC) is one of the most commonly used measurement methods for displacement and deformation of specimen surfaces in the field of experimental mechanics. Since its presentation, DIC has been evolving with many new ideas and assets. This paper proposes a new displacement field, based on Legendre polynomials and compares it with conventional second-order polynomial, commonly used in DIC's method.
\end{abstract}

\section{INTRODUCTION}

In the last decades, the experimental field of solid mechanics has grown and, with it, the need for nonintrusive measurement methods. Such methods have been developed in order to reduce costs and material usage during tests. Among the possible techniques, there are the exclusively numerical ones, like the Finite Element Method, that utilizes computational modelling to analyze mechanical behavior. Other methods seek to study the mechanical behavior in structures from a non-destructive and non-intrusive monitoring, tracking complete displacement fields of deformed materials. This kind of technique requires a lower budget compared to the traditional experiments, as they dispense the need for new sensors that would be inserted in the material. Digital Image Correlation (DIC) is one of the most used nonintrusive techniques for its practicality and its cost-benefit ratio. The method uses one or more cameras to capture images of plane surfaces under load effects. Given a pair of images as input of the process, DIC identifies a displacement field that represents the movement of surface points relative to their initial position. From the displacement data in a region, it is possible to obtain a series of information about the specimen, such as deformations, tensions, mechanical and fracture properties. According to [1], DIC is an optical metrology based on digital images processing and numerical computing and, in numerous literature actually, has been given different names, such as digital speckle correlation method (DSCM) 
[2,3], texture correlation [4], computer-aided speckle interferometry (CASI) [5, 6] and electronic speckle photography (ESP) [7, 8].

Since its presentation and mathematical formulation by [9] at the University of South Carolina, DIC has been evolving with new techniques, implementations and ideas as seen in [10]. Many studies were made to optimize the method, as in [11] and [12]. The latter being the introductory paper for Newton-Raphson method in this context. As in the scope of solid mechanics, precision is essential, many advancements about data refinement were also made [13, 14].

Some of the first experimental applications of the method analyzed rotation of a rigid body, vibration of a rod [15] and flow of a speckled fluid [16]. Beyond these, another relevant application of the DIC research is fracture characterization [17].

The method evolved to other directions beyond traditional 2D-DIC with subset approach. [18] first developed 3D-DIC (Stereo-DIC), [19] introduced a global approach (Full-field) employing finite element shape functions and, using tomography, DVC (Digital Volume Correlation) was proposed by [20].

An alternative displacement field for movement mapping that can be applied to DIC is one used in Finite-Volume Direct Averaging Micromechanics Theory (FVDAMT). FVDAMT is a recent concept of thermo-mechanical problems solver presented by [21] that utilizes the same theoretical basis of the Higher-Order Theory [22]. A formulation for the method using a Legendre Polynomial as displacement field can be seen in [23].

Although decades of advancements, there's still space left for refinement of the method. Currently, there is a gap about the available options for vectoral displace
by proposing a new mapping function, based on Lege
This paper brings the formulations of FVDMT to
menting these concepts, it is possible to make a comp through analytical models found in the literature.
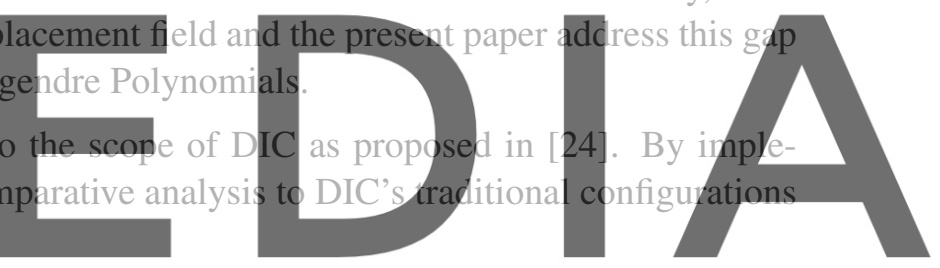

In this article, we reviewed recent literature in fields of DIC and FVDAMT. This recapitulation contex-

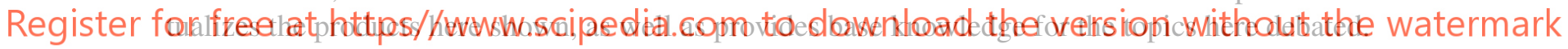

As will be seen in detail in the Section 4, a strand of the DIC method that utilizes the Legendre Polynomial as displacement field is formulated and implemented. The computer program created uses subset approach, employing the Newton-Raphson method for parameter optimization and bicubic splines for interpolation.

Following, numerical models are defined and analyzed comparatively to analytical models found in the literature for validation, as uniaxial tension. The images used as input are obtained by a Finite Elements Method computer simulation of a load effect on a surface through a plugin of itom software.

\section{DIGITAL IMAGE CORRELATION}

As seen in [19], Digital Image Correlation (DIC) is a method utilized for displacement and deformation measurement of surfaces under the effect of thermic or mechanical loads. The method utilizes pairs of digital images of a specimen under controlled position, luminosity and color, wherein the first image is taken before and the latter after the effects of the load, as seen in Figure 1. Those images are known as original and deformed, respectively, and must have speckles. 


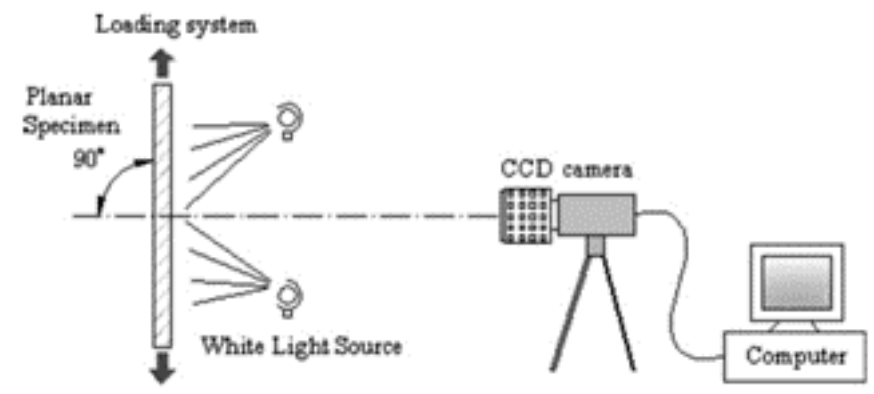

Figure 1: Optical image acquisition system for the 2D-DIC method. [1]

Numerically, the method consists in iterating a set of parameters to optimize a correlation coefficient and recalculating the displacement of given point and its neighborhood the original image relative to the deformed image [25]. The following equation shows the expression of the Sum of Squared Difference correlation coefficient (SSD)

$$
S S D=\int_{-1}^{1} \int_{-1}^{1}\left[I(x, y)-I^{\prime}(x+u(x, y), y+v(x, y))\right]^{2} d x d y
$$

where I and I' are the intensity of color functions of the original and deformed image, respectively, and $\mathrm{u}$ and $\mathrm{v}$ are the displacement field for the $\mathrm{x}$ and $\mathrm{y}$ directions measured in pixels.
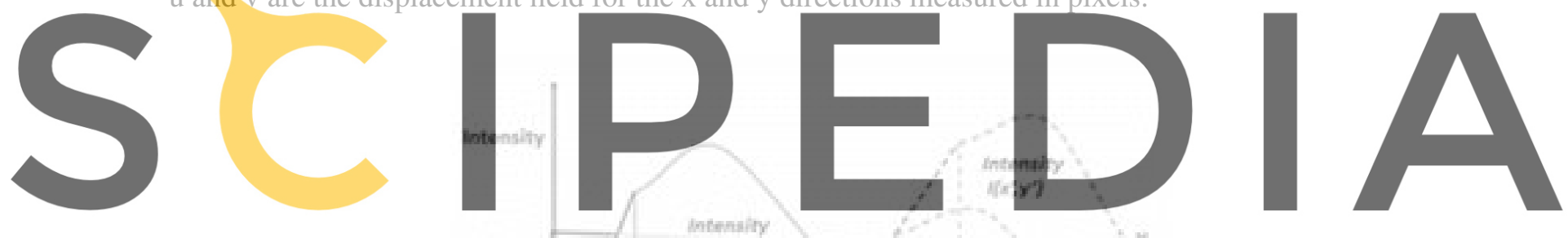

Register for free at https//www.scipedia.com to download the version without the watermark

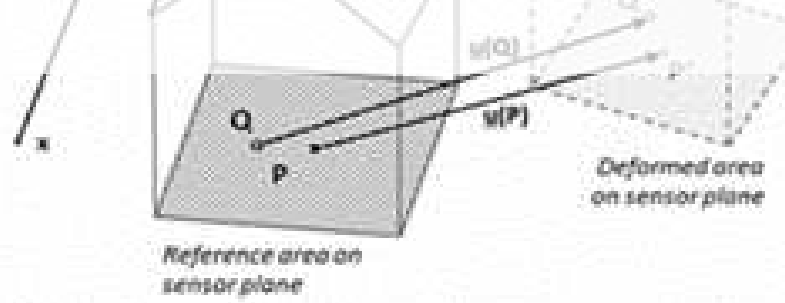

Figure 2: Schematic of the mapping process for the intensity pattern I, as the specimen is deformed. [26].

Section 4 discusses the evaluation of the displacement values iteratively estimated by the displacement field used. The correlation coefficient, on the other hand, is optimized by the Newton-Raphson method for non-linear equations system, as seen in [19]. The following equation is a matrix representation of the method:

$$
\{x\}_{n+1}=\{x\}_{n}-[J]_{n}^{-1}\{x\}_{n}, n \in \mathbb{N}
$$


where $\{x\}_{n}$ is the estimated root of a function in the nth iteration and $\mathrm{J}$ is the Jacobian matrix.

In DIC's case, $\{x\}_{n}$ is a vector composed of the unknown parameters $U_{i}$ and $V_{i}$ present in the displacement fields. The Jacobian matrix is given by:

$$
J=\nabla u
$$

or

$$
J_{i j}=\left\{\frac{\partial u_{i}}{\partial x_{j}}\right\}
$$

The algorithm developed in this study correlates the input images based on regions called cells which are defined by the user, utilizing a local approach known as Subset. Thereby, the displacement data acquired refer to the center of a cell. Thus, the image was divided in many cells along its surface for movement characterization.

\section{VECTORAL DISPLACEMENT FIELDS}

As said previously, the displacement fields are equations that characterize displacement of a given point and its neighbor region because of the load. The following equation is a second-order displacement field commonly used in DIC's scope:
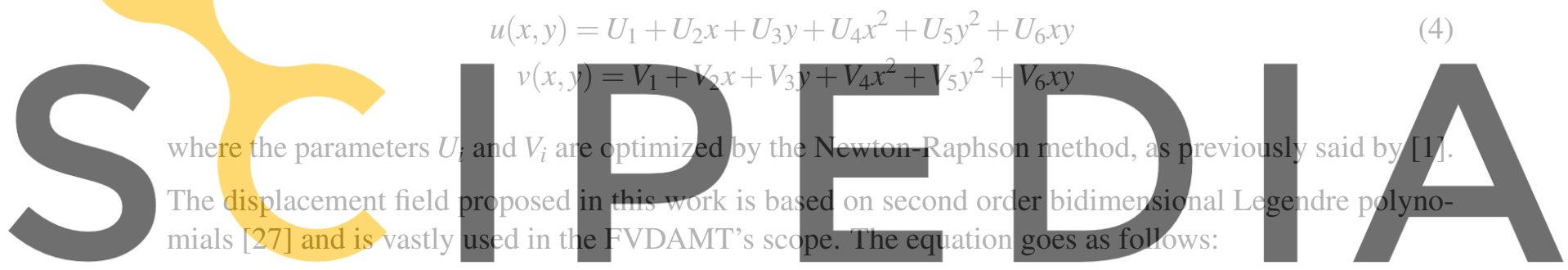

Register for free at https//www.scipedia.copn to download2the version without the watermark

$$
v(x, y)=V_{1}+x V_{2}+y V_{3}+\frac{1}{2}\left(3 x^{2}-\frac{d^{2}}{4}\right) V_{4}+\frac{1}{2}\left(3 y^{2}-\frac{h^{2}}{4}\right) V_{5}
$$

where $\mathrm{d}$ and $\mathrm{h}$ are the width and height of the region to be analyzed.

The proposal of this study is to implement a DIC algorithm capable of running both displacement fields. The mechanical and dimensional parameters are input in the Finite Elements simulation for image generation. It is possible to use these parameters to formulate an analytical function for displacement data referent to the uniaxial tension test. Data acquired in the DIC process from both displacement fields is then compared to validate accuracy and processing time. The analytical displacement data serves them as reference.

\section{RESULTS}

In this section are presented examples of Uniaxial Traction and Brazilian Test. The results obtained by the proposed formulation are compared with the polynomial solutions (Eq. 5), commonly used in DIC, and analytical to validate the results. 


\subsection{Uniaxial Tension Test}

In this example, the images were divided in grids of $17 x 17$ pixels-cells with side length of 100 pixels. Then, we evaluated displacements in the centers of the cells using three different methods. For the uniaxial tension test simulation, the following table shows the dimensional and mechanical parameters used in the software (itom):

Table 1: Mechanical and dimensional parameters for the uniaxial tension test simulation.

\begin{tabular}{cc}
\hline Tension $(\mathrm{kN} / \mathrm{mm})$ & 0.01 \\
\hline Young Modulus $\left(\mathrm{kN} / \mathrm{mm}^{2}\right)$ & 0.15 \\
\hline Poisson's Ratio & 0.3 \\
\hline Width $(\mathrm{mm})$ & 200 \\
\hline Height & 200 \\
\hline Thickness $(\mathrm{mm})$ & 1
\end{tabular}

The following figure shows the resulting the displacement data obtained from the Equation 6 under the uniaxial tension simulation:
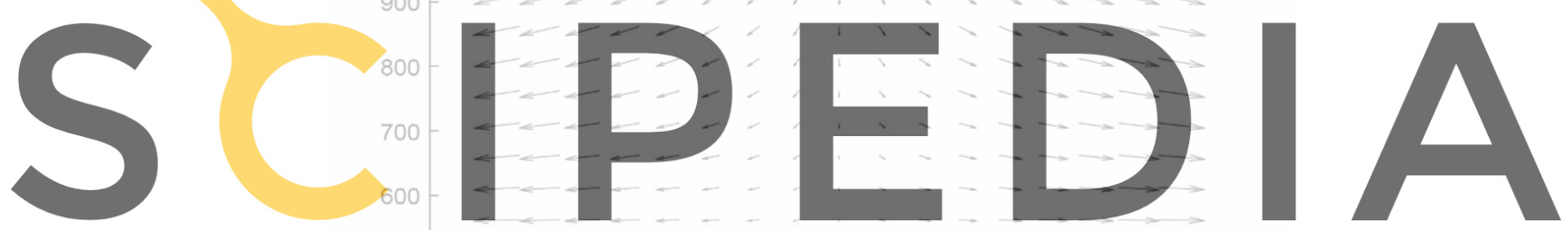

Register for free at https//www.scipediā.cöm to downlöađ the version without the watermark

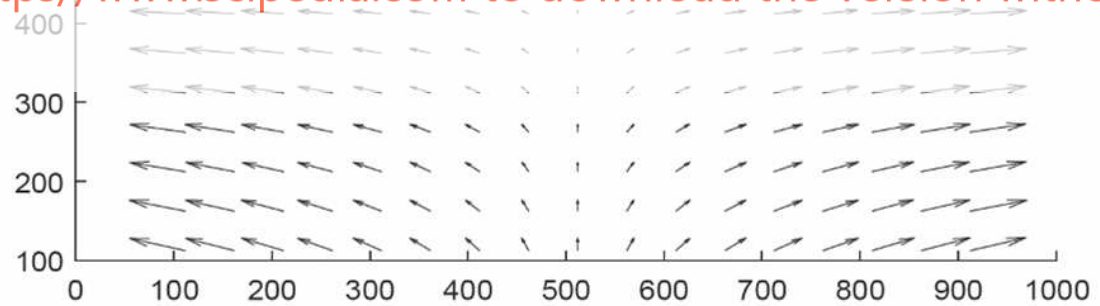

Figure 3: Displacement of the surface under the effect of uniaxial tension as estimated by the Legendre polynomial field.

where each vector represents the displacement of the center of each cell along the image. It is noticeable the surface deforming conforms to a traction along its horizontal axis.

For comparative purposes, the following figure represents the horizontal displacement along a horizontal axis of the image: 


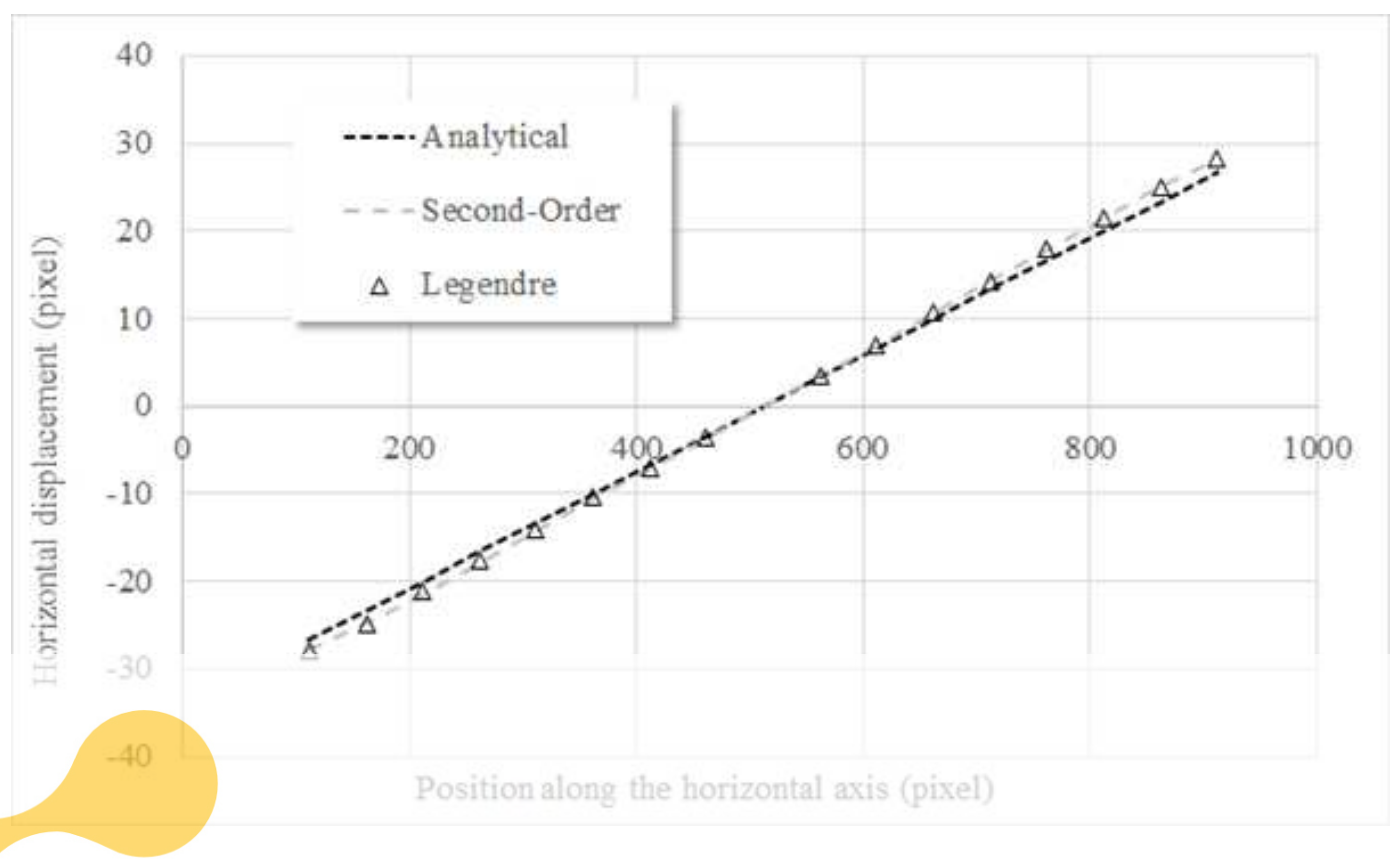

Figure 4: Horizontal displacement along a horizontal axis.

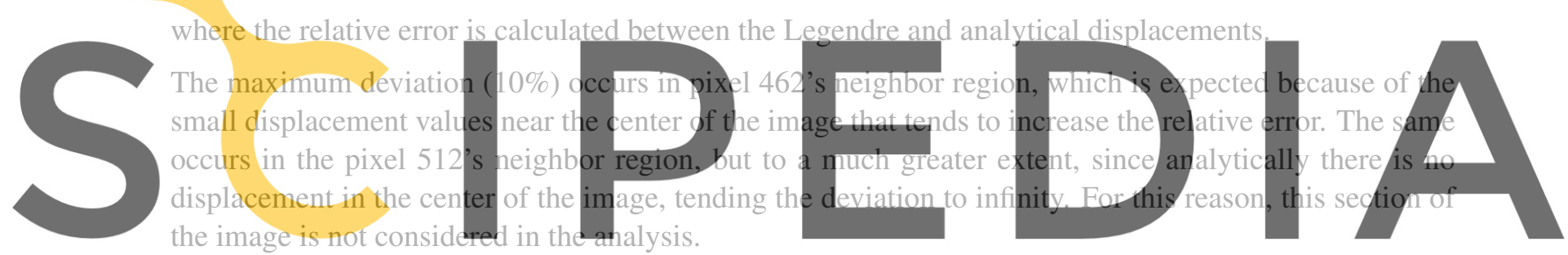
the image is not considered in the analysis.

Both displacements estimated by DIC.are close to each other along this horizontal axis. Also the LegRegister for free at htths /y wwW s Glpedia, con to download the versign without the watermark also worth noting that both numerical models work at a similar computational time.

\subsection{Brazillian Test}

In this example, a numerical model of cylindrical specimen is subjected to diametral compression loading, as shown below:

This example was chosen by the existence of the analytical solutions for the radial displacement fields, as can be seen in [28]. The study region has dimensions of $1024 \times 1024$ pixels positioned in the center of the disk. The physical properties and loading adopted in the generation of the deformed image are as follows:

The displacement fields obtained with the formulation proposed in this work were compared with the analytical solutions [28] and with the standard solution (Second-Order) of the DIC method. In Figure 6 it is possible to observe the comparison between the horizontal displacements to the horizontal line in the position 400 pixels above the central horizontal axis, with a good agreement between the approaches, re- 


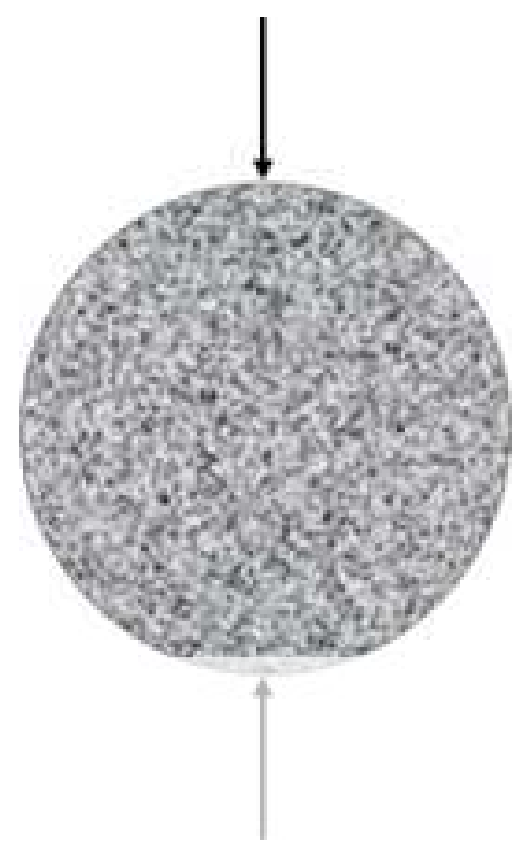

Figure 5: Brazilian test for concentrated force.

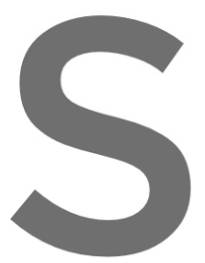

Table
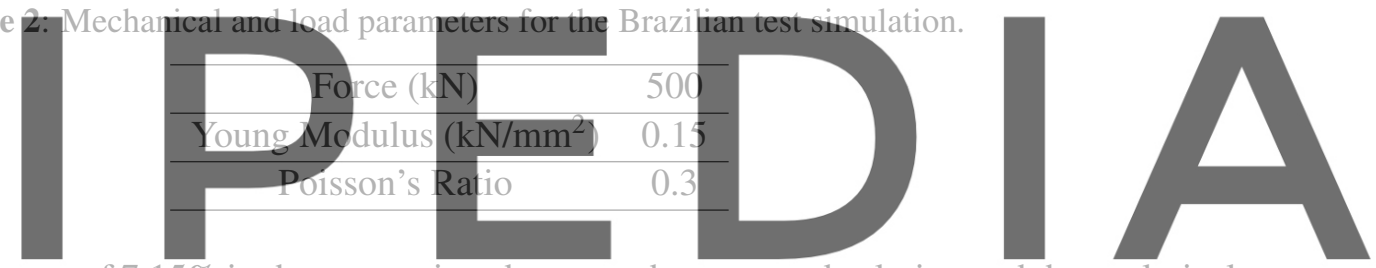

sulting in a maximum error of $7.15 \%$ in the comparison between the proposed solution and the analytical

Register for free at hointts near the edge,

Figure 7 shows the vertical displacement for the same axis studied in Figure 06. The maximum error found is $10.8 \%$ in the central position of the study line. Maximum error (4 pixels) found in middle of the image because the center region has smaller deformations, which introduces interpolation error in deformed image.

\section{CONCLUSION}

In this paper, a new strand for displacement field in DIC method was formulated and implemented. The new field is bound to the Finite-volume Direct Averaging Micromechanics Theory by the Legendre polynomial displacement field. During the development stage, we focused on studying the concepts of DIC and FVDAMT seeking for basic knowledge needed to apply those methods.

After implementation of the method and creation of analytical fields, we began the testing phase. Using itom software [29] pairs of images of a hypothetical specimen were generated. The distortion field applied models the effect of uniaxial tension. 


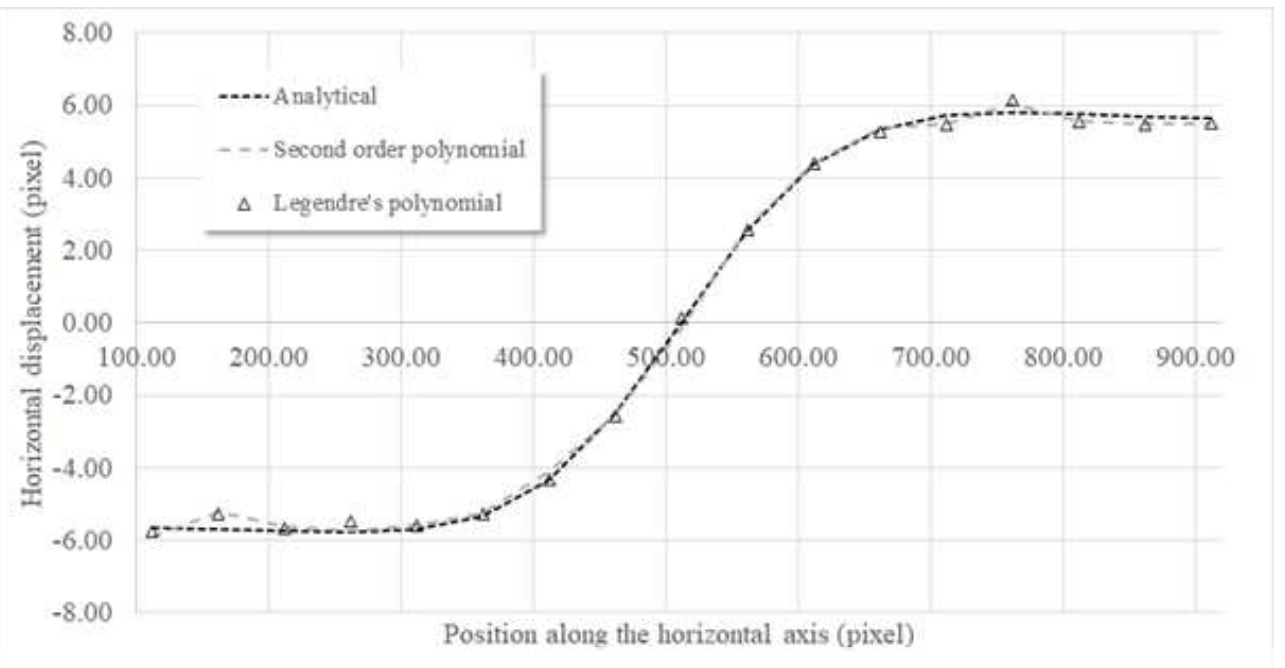

Figure 6: Horizontal displacement on the horizontal axis at 400 pixels above the center of the disk.
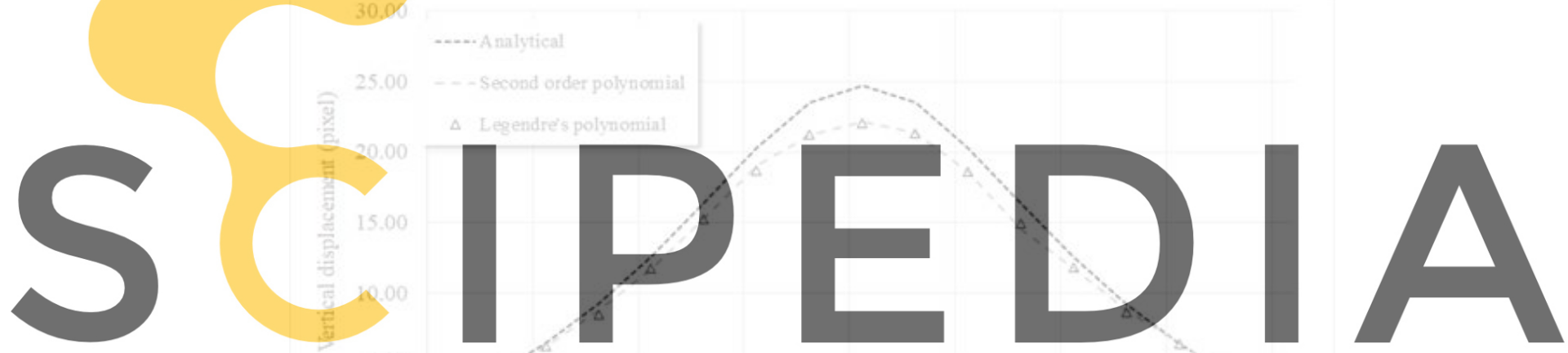

Register for free at https//www.scipedia.com to download the version without the watermark

Figure 7: Vertical displacement on the horizontal axis at 400 pixels above the center of the disk.

The images generated were used as input in the program developed applying both Legendre Polynomial and conventional polynomial as displacement fields. Also, the displacement was calculated using the analytical method.

The data acquired in this study shows that both displacements acquired via DIC are extremely close and precise, as the relative error to the analytical displacement averaged 3,4\%. This shows how DIC is a reliable method for measuring displacement and proves the applicability of the Legendre polynomials as displacement fields.

By having one more concept implemented into DIC, the users of this method have more variety of configurations. But, the most important advantage is the theoretic proximity with FVDAMT, which 
makes possible the use of its formulations for advanced mechanical analysis and characterization of the specimen.

\section{REFERENCES}

[1] Pan, B., Qian, K., Xie, H., and Asundi, A. Two-dimensional digital image correlation for in-plane displacement and strain measurement: a review. Measurement Science and Technology, (2009).

[2] Zhang, D.; Zhang, X.; Cheng, G. Compression strain measurement by digital speckle correlation. Experimental mechanics, (1999).

[3] Zhou, Peng; Goodson, Kenneth E. Subpixel displacement and deformation gradient measurement using digital image/speckle correlation. Optical Engineering, (2001).

[4] Bay, Brian K. Texture correlation: a method for the measurement of detailed strain distributions within trabecular bone. Journal of Orthopaedic Research, (1995).

[5] Chen, D. J. et al. Digital speckle-displacement measurement using a complex spectrum method. Applied optics, (1993).

[6] Gaudette, Glenn R. et al. Computer aided speckle interferometry: a technique for measuring deformation of the surface of the heart. Annals of Biomedical Engineering, (2001).

[7] Sjödahl, Mikael; Benckert, L. R. Electronic speckle photography: analysis of an algorithm giving the displacement with subpixel accuracy. Applied Optics, (1993).
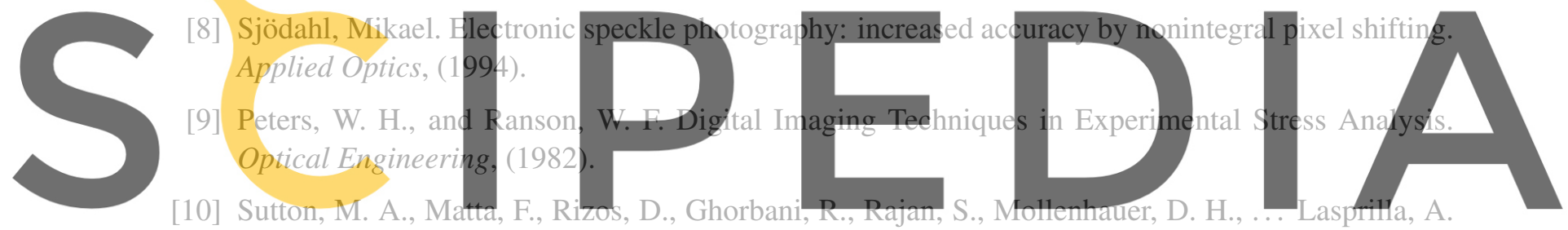
O.. Recent Progress in Digital Image Correlation: Background and Developments since the 2013

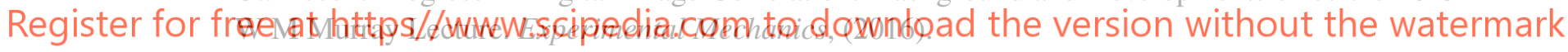

[11] Sutton, M., Mingqi, C., Peters, W., Chao, Y., and McNeill, S. Application of an optimized digital correlation method to planar deformation analysis. Image and Vision Computing, (1986).

[12] Bruck, H. A., McNeill, S. R., Sutton, M. A., and Peters, W. H.. Digital image correlation using Newton-Raphson method of partial differential correction. Experimental Mechanics, (1989).

[13] Schreier, Hubert W.; Braasch, Joachim R.; Sutton, Michael A. Systematic errors in digital image correlation caused by intensity interpolation. Optical Engineering, (2000).

[14] Schreier, H. W., and Sutton, M. A. . Systematic errors in digital image correlation due to undermatched subset shape functions. Experimental Mechanics, (2002).

[15] Peters, W. H., Ranson, W. F., Sutton, M. A., Chu, T. C., and Anderson, J. . Application of Digital Correlation Methods To Rigid Body Mechanics. Optical Engineering, (1983).

[16] He, Z. H., Sutton, M. A., Ranson, W. F., and Peters, W. H. Two-dimensional fluid-velocity measurements by use of digital-speckle correlation techniques. Experimental Mechanics, (1984). 
[17] Chu, T. C., Ranson, W. F., Sutton, M. A. Applications of digital-image-correlation techniques to experimental mechanics. Experimental mechanics, (1985).

[18] Luo, P.F., Chao, Y.J., Sutton, M.A. et al. Accurate measurement of three-dimensional deformations in deformable and rigid bodies using computer vision. Experimental Mechanics, (1993).

[19] Sun, Y., Wong, C. K., Su, F., and Pang, J. H. L. Finite element formulation for a digital image correlation method. Applied Optics, (2005).

[20] Bay, B.K., Smith, T.S., Fyhrie, D.P. et al. Digital volume correlation: Three-dimensional strain mapping using X-ray tomography. Experimental Mechanics, (1999).

[21] Bansal, Y., Zhong, Y., and Pindera, M.-J. Efficient reformulation of the higher-order theory for FGMs. Materials Science Forum, (2003).

[22] Aboudi, J., Pindera, M. J., and Arnold, S. M. Higher-order theory for functionally graded materials. Composites Part B: Engineering, (1999).

[23] Cavalcante, M. A. A., and Pindera, M. Generalized FVDAM theory for elastic e plastic periodic materials. International Journal of Plasticity, (2016).

[24] Cavalcante, M. A. A. Modelling of the transient thermo-mechanical behavior of composite material structures by the finite-volume theory. Diss. MS Thesis, Federal University of Alagoas, Maceio, Alagoas, Brazil, 2006.

[25] Varady Filho, C. A. F. V. Um ambiente computacional de alto desempenho para cálculo de deslo-

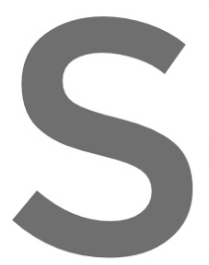
camentos usando Alagoas, (2016).

[26] Sutton, M. A. Cornputer Vision-Based Nonc A Generational Transformation. Applied Mechar
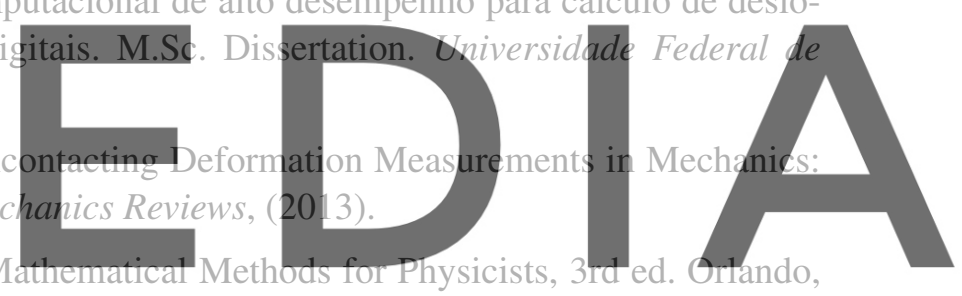

FL: Academic Press, (1985).

Register for free at https//www.scipedia.com to download the version without the watermark cular disks subjected to partially distributed compressions. International Journal of Mechanical Sciences, (2008).

[29] M. Gronle, W. Lyda, M. Wilke, C. Kohler, and W. Osten, itom: an open source metrology, automation, and data evaluation software. Applied Optics, (2014) 